\title{
Differential p16 expression levels in the liver, hepatocytes and hepatocellular cell lines
}

\author{
Barbara Kramar ${ }^{1}$, Dušan Šuput ${ }^{1}$, Irina Milisav ${ }^{\text {Corresp. } 1,2}$ \\ 1 Faculty of Medicine, Institute of Pathophysiology, University of Ljubljana, Ljubljana, Slovenia \\ 2 Faculty of Health Sciences, Laboratory of oxidative stress research, University of Ljubljana, Ljubljana, Slovenia \\ Corresponding Author: Irina Milisav \\ Email address: irina.milisav@mf.uni-lj.si
}

Background. One of the most frequently deleted genes in cancer is CDKN2A encoding p16. This protein is often overexpressed in senescent cells, while its suppression can bypass the oncogene-induced senescence to enable transformation and tumorigenesis. The roles of the protein p16 are recently being expanded from the cell cycle progression regulator to the cellular regulator interacting in several different pathways. Yet data on its liver and liver cells' expression are inconclusive. Methods. The expression of the p16 gene in liver and liver cells was determined by RT-qPCR and compared to its protein amounts by western blotting. Results. p16 is expressed at low levels in the liver and rat hepatocytes. Its expression varies from none to the considerable levels in the examined hepatocellular carcinoma cell lines ( $\mathrm{FaO}$ and $\mathrm{HepG}$ ) and in immortalized mouse hepatocytes. Such significant expression differences of an important cellular regulator warrant the need to closely examine the differences in biochemical pathways correlated with the p16 expression when using hepatocytes and hepatoma liver models. 


\section{Differential p16 expression levels in the liver, hepatocytes and 2 hepatocellular cell lines}

3

4

5

6

8

9

10

11

12

13

14

15

16

17

18
Barbara Kramar ${ }^{1}$, Dušan Šuput², Irina Milisav¹,2

${ }^{1}$ University of Ljubljana, Faculty of Medicine, Institute of Pathophysiology, Zaloska 4, Ljubljana, Slovenia

${ }^{2}$ University of Ljubljana, Laboratory of oxidative stress research, Faculty of Health Sciences, Zdravstvena pot 5, Ljubljana, Slovenia

Corresponding Author:

Irina Milisav ${ }^{1}$

University of Ljubljana, Faculty of Medicine, Institute of Pathophysiology, Zaloska 4, 1000 Ljubljana, Slovenia

Email address: irina.milisav@mf.uni-lj.si 


\section{Abstract}

20 Background. One of the most frequently deleted genes in cancer is CDKN2A encoding $\mathrm{p} 16$. This protein 21 is often overexpressed in senescent cells, while its suppression can bypass the oncogene-induced 22 senescence to enable transformation and tumorigenesis. The roles of the protein p16 are recently being 23 expanded from the cell cycle progression regulator to the cellular regulator interacting in several 24 different pathways. Yet data on its liver and liver cells' expression are inconclusive.

25 Methods. The expression of the $p 16$ gene in liver and liver cells was determined by RT-qPCR and 26 compared to its protein amounts by western blotting.

27 Results. $p 16$ is expressed at low levels in the liver and rat hepatocytes. Its expression varies from none 28 to the considerable levels in the examined hepatocellular carcinoma cell lines (FaO and HepG2) and in 29 immortalized mouse hepatocytes. Such significant expression differences of an important cellular 30 regulator warrant the need to closely examine the differences in biochemical pathways correlated with 31 the $p 16$ expression when using hepatocytes and hepatoma liver models.

32

33 
35

36

37

38

39

40

41

42

43

44

45

46

47

48

49

50

51

52

53

54

55

56

57

58

59

60

61

62

63

64

65

66

67

68

69

70

71

72

73

74

75

76

\section{Introduction}

Cyclin-dependent kinase inhibitor 2A (CDKN2A) gene encodes two protein regulators of cell cycle regulatory pathways, the $p 16(I N K 4 A$ or $p 16)$ and $p 14(A R F)$, which are encoded in alternative reading frames (Stone et al. 1995). Protein p16 is a regulator of cellular homeostasis controlling growth, senescence, apoptosis and cellular differentiation. It inhibits cell cycle progression through the cyclindependent kinase-4 and 6 / retinoblastoma (CDK4/6/Rb) pathway (Tyagi et al. 2017). It is an inhibitor of a cyclin-dependent kinase-4 (CDK4) (Angelico et al. 2021). This inhibition maintains the retinoblastoma protein ( $\mathrm{pRb}$, a product from the Rb gene) in a hypo-phosphorylated state and prevents the cell cycle progression from $\mathrm{G} 1$ to $\mathrm{S}$ phase (Li et al. 2011).

In addition to this canonical pathway, several retinoblastoma-independent pathways of $\mathrm{p} 16$ have been described recently. The examples include (1) regulation of nucleotide biosynthesis involving a pentose phosphate pathway enzyme ribose 5-phosphate isomerase A (Buj et al. 2019). (2) A direct interaction of p16 with the p65 subunit of the NF- $\kappa B$ complex can decrease the NF- $\kappa B$-promoted tumorigenesis in the absence of the inhibitor IKB $\alpha$ (Wolff \& Naumann 1999). (3) p16 can bind and inhibit JNK1/3 by binding to the c-jun binding site to impair the activation of AP-1 (Choi et al. 2005). (4) It also impairs the transcription factor eEF1A2 translational activity (Lee et al. 2013). Further functions involve (5) oxidative stress decrease (Jenkins et al. 2011) and (6) mitochondrial biogenesis (Tyagi et al. 2017).

Protein p16 is expressed in normal tissues and in solid tumours (Angelico et al. 2021). Its non-cancerous tissue expression is mostly low to moderate according to the Human Protein Atlas data (Atlas.)(CDKN2A), with high expression only in glandular cells of the colon and in late spermatids. Nevertheless, the reduced amounts of p16 tumour suppressor enable transformation and tumorigenesis (Buj et al. 2019). Therefore, its role has been mostly investigated in cancer and ageing (Buj \& Aird 2019). p16 reduction is linked to different types of cancers, e.g. melanoma, lymphoma, pancreatic adenocarcinoma, non-small cell lung cancer, gastric cancer, colorectal cancer, etc. (Zhao et al. 2016).

Loss of p16 expression because of promoter methylation was reported in $40 \%$ of the investigated cell lines in one study (Murai et al. 2005) and it also commonly occurs in hepatocellular carcinoma (HCC) (Lv et al. 2017). This is a prevalent malignancy in Asia (Lv et al. 2017), the fifth most common malignant disease in men worldwide and the cause of the second most common cancer-related deaths in men (Torre et al. 2015). The authors of a meta-analysis reported that p16 promoter methylation in HCC was increased with age and hepatitis virus B and C infections (Lv et al. 2017). Nevertheless, p16 can be expressed or not in HCC and examination of the p16 status along with another tumour suppressor p27 was proposed as a more accurate tool for predicting the prognosis of HCC (Matsuda \& Ichida 2006). Other intragenic mutations of the p16 gene were also reported in HCC in addition to the promoter methylation (Kita et al. 1996). No p16 expression is reported for human hepatocytes so far (Atlas.)(CDKN2A), although p16 was reported as one of the senescent markers in old mice on a high-fat diet (Ogrodnik et al. 2017). Low liver expression of p16 in human liver cholangiocytes, but not in hepatocytes, is described (Atlas.)(CDKN2A) and a p16 hepatocyte expression was detected by immunohistochemistry in the cases of advanced liver fibrosis (Csepregi et al. 2008).

Here we describe the differences in p16 expression levels among liver, primary and immortalized hepatocytes and hepatoma cell lines and discuss the implications of expression differences of this potentially important regulator of several cellular pathways.

Peer) reviewing PDF | (2021:07:63307:1:1:NEW 15 Sep 2021) 


\section{Materials \& Methods}

78

79

80

81

82

83

84

85

86

87

88

89

90

91

92

93

94

95

96

97

98

99

100

101

102

103

104

105

106

107

108

109

110

111

112

113

114

115

116

117

118

119

120

121

122

123

124

125

All reagents were purchased from Sigma-Aldrich (Merck), unless otherwise stated.

\section{Ethical statement}

Primary hepatocytes and the liver were isolated from adult male rats (Wistar-Hannover, Ljubljana, Slovenia, 180-280 g), ethical code numbers are U34401-44/2014/8 and U34401-21/2020/4, issued by Administration of the Republic of Slovenia for Food Safety, Veterinary Sector and Plant Protection.

\section{Cell cultures}

All cell models were grown at $37{ }^{\circ} \mathrm{C}$ in a humidified atmosphere with $5 \% \mathrm{CO}_{2}$. The rat hepatoma cell line $\mathrm{FaO}$ (ECACC, 89042701) was grown as previously described (Pirc Marolt et al. 2020). They were seeded in 6-well cell culture plates ( 650.000 cells/well) and grown for 48 hours before harvest.

The human hepatoma cell line HepG2 (ATCC, HB-8065) was grown in Dulbecco's Modified Eagle's Medium (DMEM; Gibco, 11966) with $2 \mathrm{~g} / \mathrm{L} \mathrm{D-(+)-glucose} \mathrm{(G6152),} \mathrm{supplemented} \mathrm{with} 10 \%$ FBS and $1 \%$ Pen-Strep for 48 hours before harvest. 6 -well cell culture plates ( 500.000 cells/well) were used for the seeding.

The human embryonic kidney cell line 293T (ECACC, 12022001) is a single-cell clone of regular 293 cells. The cells were seeded in 6-well cell culture plates ( 200.000 cells/well) and grown in high glucose DMEM (4.5 g/L glucose + L-glutamine, 11965-092), $10 \%$ FBS and $1 \%$ Pen-Strep for 48 hours before harvest.

Immortalized mouse neonatal hepatocytes provided by Dr Angela M. Valverde (Gonzalez-Rodriguez et al. 2009; Pardo et al. 2015) were grown in T25 cell culture flasks (seeded 100.000 cells/flask) in a high glucose DMEM (4.5 g/L glucose + L-glutamine, 11965-092), $10 \%$ FBS and $1 \%$ Pen-Strep for 48 hours before harvest.

Liver from 12-week old Wistar rats and primary hepatocytes from 8-week old Wistar-Hannover rats were isolated under ethical code numbers stated above. Reverse two-step perfusion with collagenase (C5138) was used to isolate primary hepatocytes (Nipic et al. 2010). Hepatocyte viability was at least 90 $\%$, as determined by Trypan blue stain $0.4 \%$ (Gibco, 15250). The cells were seeded on the collagen type I (C867) coated surface of 6 well plates at 500.000 cells/well for four hours in Williams medium E (W4125) supplemented with $10 \%$ FBS, $1 \%$ Pen-Strep and insulin $(0.1 \mathrm{U} / \mathrm{mL}$; I1882). For the remaining 68 hours until harvest, hepatocytes were in the Williams medium E with $0.03 \%$ bovine serum albumin (A2153), $0.5 \%$ Pen-Strep, insulin $(0.1 \mathrm{U} / \mathrm{mL})$ and $1 \mu \mathrm{M}$ hydrocortisone-21 hemisuccinate $(\mathrm{H} 2270)$. The liver were processed as previously described (Banic et al. 2011).

\section{RNA isolation and reverse-transcription quantitative polymerase chain reaction analysis (RT-qPCR)}

Total RNA was isolated with TRI reagent (T9424) and reverse transcribed using the High capacity cDNA reverse transcription kit (Applied Biosystems, 4368814) with added RNase inhibitor (Applied Biosystems, N8080119). PCR reactions ( $\leq 100 \mathrm{ng}$ CDNA/reaction) were run in duplicates using TaqMan Universal Master Mix II, with uracil-N-glycosylase (ThermoFisher Scientific, 4440038) and quantitated using the 7500 Real-Time PCR System with SDS software (v1.3.1, Applied Biosystems) or on QuantStudio 3 RealTime PCR System with Design and Analysis 2.5.0. software (ThermoFisher Scientific). The software was used to set the baseline (auto) and to determine the cycle threshold ( $\mathrm{Ct}$ ). The following TaqMan probes labelled with the FAM dye (ThermoFisher Scientific) were used: rat p16 (Rn00580664_m1), mouse p16 (Mm00494449_m1) and human p16 (Hs00923894_m1). 18S (Hs99999901_s1) was used as a reference gene.

Peer] reviewing PDF | (2021:07:63307:1:1:NEW 15 Sep 2021) 
126

127

128

129

130

131

132

133

134

135

136

137

138

139

140

141

142

143

144

145

146

147

148

149

150

151

152

153

154

155

156

157

158

159

160

161

162

163

164

165

166

167

168

169

\section{Immunoblotting}

Whole cell lysates in a lysis buffer (20 mM Hepes-KOH (H3375), pH 7.9, $125 \mathrm{mM} \mathrm{NaCl}$ (Fluka, 31434), 1 mM EDTA (E6758), 1 \% Igepal CA-360 (I8896), $10 \mathrm{mM}$ sodium pyrophosphate (P8010), $5 \mathrm{mM}$ sodium fluoride (S6776), $5 \mathrm{mM} \beta$-glycerol phosphate (50020), $0.2 \mathrm{mM}$ sodium orthovanadate (450243), $1 \mathrm{mM}$ phenylmethylsulfonyl fluoride (PMSF, P7626) and 1mM protease inhibitor cocktail (P8340) were added to the reducing Laemmli-buffer (0.25 M Tris pH 6.8, 8\% SDS, 40\% glycerol, $0.03 \%$ bromophenol blue), denatured at $95{ }^{\circ} \mathrm{C}$ for $5 \mathrm{~min}$ and $40 \mu \mathrm{g}$ of a sample was applied on $16 \%(\mathrm{w} / \mathrm{v}$ ) acrylamide gels to be separated by standard sodium dodecyl sulfate-polyacrylamide gel electrophoresis (SDS-PAGE) and blotted onto PVDF membrane (Immobilon-P, Merck-Millipore, Darmstadt, Germany). Two antibodies against p16 were used: the rabbit polyclonal (PA5-20379, Invitrogen, Thermo Fisher Scientific, Waltham, MA, USA) and the mouse monoclonal Anti-CDKN2A/p16INK4a Antibody (F-12; sc-1661, Santa Cruz Biotechnology Inc., Dallas, TX, USA). The signal was detected by luminescence through the secondary goat anti-rabbit and anti-mouse antibodies, respectively, conjugated to horseradish peroxidase (Bio-Rad, Hercules, CA, USA), visualized using Fusion FX imager (Vilber, Marne-la-Vallée, France) and quantified by densitometry using Image Studio Lite software (LI-COR, Lincoln, USA).

\section{Statistical analysis}

All data are presented as means \pm SD. The number of biological replicates is stated in the figure legends. GraphPad Prism 9.1.2 with an inbuilt algorithm to test the equality of variances from medians, the Brown-Forsythe test, was used for statistical analysis. In the case of equal variances, one-way ANOVA was used and Tukey's multiple comparisons test.

\section{Results}

Expression of the $p 16$ gene was determined in rat liver, hepatocytes and hepatocyte-derived cells (Figure 1) and compared to the expression in HEK 293T cell line with reported $p 16$ expression (Cui et al. 2015). Low levels of $p 16$ expression were detected in the liver and primary rat hepatocytes. Somewhat more transcript was in HepG2 cells. Comparable amounts of $p 16$ were transcribed from immortalized hepatocytes and HEK 293T cell line (Table 1). In contrast to the p16 expression in HepG2, there was no expression whatsoever in the $\mathrm{FaO}$ hepatoma cell line.

The expression of $18 \mathrm{~S}$ RNA served as a cDNA quality control. The mean threshold cycle (Ct) values of the reference gene are similar; the arithmetic mean +/- SD is $14.39+/-0.29$. The histograms of delta $\mathrm{Ct}(\Delta \mathrm{Ct})$ values, therefore, follow an identical pattern to that of the $\mathrm{Ct}$ values with statistically significant differences between all hepatocyte cell lines (Figure 2).

In conclusion, similar gene expression was measured in primary hepatocytes of young (pre-sexually matured) rats and in the liver of adult rats. The p16 expression markedly differed among the two hepatoma cell lines and immortalized hepatocytes, in which the expression ranged from 0 to well expressed, with the $\mathrm{Ct}$ number around 24 and $\Delta \mathrm{Ct}$ around 10 (Figure 2).

Gene expression data were then compared with the protein levels. Amounts of p16 protein were visualized by two commercial antibodies, a polyclonal (Figure 3) and a monoclonal (Supplemental Figure 1). Both detected many bands of different sizes in all tested liver cells and tissue, however, the bands running close to a $17 \mathrm{kDa}$ protein marker closely resembled a DNA expression pattern. The relative differences in protein amounts between the hepatic cells and tissue follow the gene expression pattern of large statistically significant differences (Figure 3).

Peer) reviewing PDF | (2021:07:63307:1:1:NEW 15 Sep 2021) 


\section{Discussion}

We found large differences in expression levels of the $p 16$ gene in the liver and various closely related hepatic cells that are widely used as experimental models. Unlike the expression levels of a reference gene that was stable, the expression of $p 16$ ranged from zero to substantial levels. The protein amounts of positive control and hepatic cell lines and tissue closely resemble the gene expression levels when the bands that run close to the $17 \mathrm{kDa}$ protein marker are compared. No strong p16 band was observed, which is in line with the Protein Atlas antibody data (Atlas.). Many bands of different sizes are highlighted when the blots are probed with a polyclonal or monoclonal antibody, which together with a weak signal warrant for the development of an antibody suitable for detection of baseline levels of p16. Post-transcriptional modifications of p16 may account for a band shift in IMHP. Indeed, a p16 protein can be phosphorylated at four sites and also methylated (Gump et al. 2003; Jiao et al. 2018). The appropriate size of protein detected in the positive control and hepatic samples as well as the relative amounts of various hepatic cells and the tissue are in line with the gene expression data and therefore strengthen the protein band identification. This process also highlights difficulties in comparing the gene expression with the protein data reported by many authors (Csardi et al. 2015; Liu et al. 2016; Pascal et al. 2008).

187

No p16 protein has been detected in hepatocytes according to the Protein Atlas(Atlas.) (CDKN2A). Nevertheless, p16 has a role in hepatocellular carcinoma and steatosis progression (see below). Overexpression of 16 gene was reported to inhibit proliferation and reduce invasion ability of hepatocellular carcinoma (Huang et al. 2003). Therefore, these differences in expression imply a need to evaluate $p 16$ expression in every experimental liver model to establish whether metabolic differences in related cell models are due to variations in $p 16$ expression.

A correlation was found between the p16 expression and hepatic fat accumulation since the removal of p16 expressing senescent cells in a mice model reduced steatosis (Ogrodnik et al. 2017). In approximately 20-30 \% of people liver steatosis progresses to a harmful nonalcoholic steatohepatitis characterized by liver inflammation, dysfunctional fibrosis, and hepatocyte death (Rinella 2015). No p16 was detected by immunohistochemistry in human non-cancer liver tissue without fibrosis, while there

200

201

202

203

204

205

206

207

208

209

210 was a significant p16 expression in the case of advanced liver fibrosis (Csepregi et al. 2008). Liver fibrosis develops as a result of hepatic stellate cells (HSCs) activation to myofibroblasts (MFBs), which deposit collagen during hepatic fibrogenesis also to replace the apoptotic hepatocytes (Sancho et al. 2012). Transforming growth factor-beta (TGF- $\beta$ ) level increases during the development of liver fibrosis and induces apoptosis in hepatocytes, while also contributes to the activation of HSCs (Proell et al. 2007; Sanchez et al. 1996).

Escaping from the TGF- $\beta$ suppression is also a prerequisite for liver tumour progression, as TGF- $\beta$ also activates the survival signals (Caja et al. 2007). This process is important in liver carcinogenesis. TGF- $\beta$ was reported as a suppressor factor for adult quiescent hepatocytes, but not for FaO hepatoma cells, where it had two roles, both suppressing and promoting carcinogenesis (Caja et al. 2007). These are the 
211 two cell models in which we have determined a different expression of $p 16$. It fits with the notion that 212 there is an overexpression of p16 in senescent cells, while its suppression can bypass the oncogene-

213 induced senescence to enable transformation and tumorigenesis (Buj et al. 2019).

214

\section{Conclusions}

216 As p16 can regulate several pathways and is differentially expressed in closely related hepatocytes and 217 hepatocyte-derived cells, it is necessary to check for the possible differences in biochemical pathways in 218 hepatocyte liver models that may arise because of the differences in p16 expression.

219

220

221

222

223

224

225

226

227

228

229

230

231

232

233

234

235

236

237

238

239

240

241

242

243

244

245

246

247

248

249

250

251

252

253

254

255

256

\section{Acknowledgements}

Izak Patrik Miller and Patrik Prša kindly assisted with liver processing. We are grateful to Dr Ángela M. Valverde, Instituto de Investigaciones Biomedicas 'Alberto Sols', CSIC, Madrid, Spain for a kind donation of mouse immortalized hepatocytes and to Dr Maria Monsalve for the HEK 293T cells through the H2020-MSCA-ITN:721236 TREATMENT project.

\section{References}

Angelico G, Santoro A, Inzani F, Straccia P, Spadola S, Arciuolo D, Valente M, D'Alessandris N, Benvenuto R, Travaglino A, Raffone A, and Zannoni GF. 2021. An Emerging Anti-p16 Antibody-BC42 Clone as an Alternative to the Current E6H4 for Use in the Female Genital Tract Pathological Diagnosis: Our Experience and a Review on p16ink4a Functional Significance, Role in Daily-Practice Diagnosis, Prognostic Potential, and Technical Pitfalls. Diagnostics (Basel) 11. 10.3390/diagnostics11040713

Atlas. THP. The Human Protein Atlas. CDKN2A. Available at https://www.proteinatlas.org/ENSG00000147889CDKN2A/tissue (accessed May 16, 2021.

Banic B, Nipic D, Suput D, and Milisav I. 2011. DMSO modulates the pathway of apoptosis triggering. Cell Mol Biol Lett 16:328-341. 10.2478/s11658-011-0007-y

Buj R, and Aird KM. 2019. p16: cycling off the beaten path. Mol Cell Oncol 6:e1677140. 10.1080/23723556.2019.1677140

Buj R, Chen CW, Dahl ES, Leon KE, Kuskovsky R, Maglakelidze N, Navaratnarajah M, Zhang G, Doan MT, Jiang H, Zaleski M, Kutzler L, Lacko H, Lu Y, Mills GB, Gowda R, Robertson GP, Warrick JI, Herlyn M, Imamura Y, Kimball SR, DeGraff DJ, Snyder NW, and Aird KM. 2019. Suppression of p16 Induces mTORC1-Mediated Nucleotide Metabolic Reprogramming. Cell Rep 28:1971-1980 e1978. 10.1016/j.celrep.2019.07.084

Caja L, Ortiz C, Bertran E, Murillo MM, Miro-Obradors MJ, Palacios E, and Fabregat I. 2007. Differential intracellular signalling induced by TGF-beta in rat adult hepatocytes and hepatoma cells: implications in liver carcinogenesis. Cell Signal 19:683-694. 10.1016/j.cellsig.2006.09.002

Choi BY, Choi HS, Ko K, Cho YY, Zhu F, Kang BS, Ermakova SP, Ma WY, Bode AM, and Dong Z. 2005. The tumor suppressor p16(INK4a) prevents cell transformation through inhibition of c-Jun phosphorylation and AP-1 activity. Nat Struct Mol Biol 12:699-707. 10.1038/nsmb960

Csardi G, Franks A, Choi DS, Airoldi EM, and Drummond DA. 2015. Accounting for experimental noise reveals that mRNA levels, amplified by post-transcriptional processes, largely determine steady-state protein levels in yeast. PLoS Genet 11:e1005206. 10.1371/journal.pgen.1005206

Csepregi A, Röcken C, Ebert M, and P. M. 2008. p16 protein expression in the liver: an immunohistochemical study. Zeitschrift für Gastroenterologie 46:P2_45. 10.1055/s-2008-1037553

Cui C, Gan Y, Gu L, Wilson J, Liu Z, Zhang B, and Deng D. 2015. P16-specific DNA methylation by engineered zinc finger methyltransferase inactivates gene transcription and promotes cancer metastasis. Genome Biol 16:252. 10.1186/s13059-015-0819-6 
257

258

259

260

261

262

263

264

265

266

267

268

269

270

271

272

273

274

275

276

277

278

279

280

281

282

283

284

285

286

287

288

289

290

291

292

293

294

295

296

297

298

299

300

301

302

303

304

305

306

307

308
Gonzalez-Rodriguez A, Alba J, Zimmerman V, Kozma SC, and Valverde AM. 2009. S6K1 deficiency protects against apoptosis in hepatocytes. Hepatology 50:216-229. 10.1002/hep.22915

Gump J, Stokoe D, and McCormick F. 2003. Phosphorylation of p16INK4A correlates with Cdk4 association. J Biol Chem 278:6619-6622. 10.1074/jbc.C200622200

Huang JZ, Xia SS, Ye QF, Jiang HY, and Chen ZH. 2003. Effects of p16 gene on biological behaviours in hepatocellular carcinoma cells. World J Gastroenterol 9:84-88. 10.3748/wjg.v9.i1.84

Jenkins NC, Liu T, Cassidy P, Leachman SA, Boucher KM, Goodson AG, Samadashwily G, and Grossman D. 2011. The p16(INK4A) tumor suppressor regulates cellular oxidative stress. Oncogene 30:265-274. 10.1038/onc.2010.419

Jiao Y, Feng Y, and Wang X. 2018. Regulation of Tumor Suppressor Gene CDKN2A and Encoded p16-INK4a Protein by Covalent Modifications. Biochemistry (Mosc) 83:1289-1298. 10.1134/S0006297918110019

Kita R, Nishida N, Fukuda Y, Azechi H, Matsuoka Y, Komeda T, Sando T, Nakao K, and Ishizaki K. 1996. Infrequent alterations of the p16INK4A gene in liver cancer. Int J Cancer 67:176-180. 10.1002/(SICI)10970215(19960717)67:2<176::AID-IJC4>3.0.CO;2-Q

Lee MH, Choi BY, Cho YY, Lee SY, Huang Z, Kundu JK, Kim MO, Kim DJ, Bode AM, Surh YJ, and Dong Z. 2013. Tumor suppressor p16(INK4a) inhibits cancer cell growth by downregulating eEF1A2 through a direct interaction. J Cell Sci 126:1744-1752. 10.1242/jcs.113613

Li J, Poi MJ, and Tsai MD. 2011. Regulatory mechanisms of tumor suppressor P16(INK4A) and their relevance to cancer. Biochemistry 50:5566-5582. 10.1021/bi200642e

Liu Y, Beyer A, and Aebersold R. 2016. On the Dependency of Cellular Protein Levels on mRNA Abundance. Cell 165:535-550. 10.1016/j.cell.2016.03.014

Lv X, Ye G, Zhang X, and Huang T. 2017. p16 Methylation was associated with the development, age, hepatic viruses infection of hepatocellular carcinoma, and p16 expression had a poor survival: A systematic meta-analysis (PRISMA). Medicine (Baltimore) 96:e8106. 10.1097/MD.0000000000008106

Matsuda Y, and Ichida T. 2006. p16 and p27 are functionally correlated during the progress of hepatocarcinogenesis. Med Mol Morphol 39:169-175. 10.1007/s00795-006-0339-2

Murai Y, Hayashi S, Takahashi H, Tsuneyama K, and Takano Y. 2005. Correlation between DNA alterations and p53 and p16 protein expression in cancer cell lines. Pathol Res Pract 201:109-115. 10.1016/j.prp.2005.01.002

Nipic D, Pirc A, Banic B, Suput D, and Milisav I. 2010. Preapoptotic cell stress response of primary hepatocytes. Hepatology 51:2140-2151. 10.1002/hep.23598

Ogrodnik M, Miwa S, Tchkonia T, Tiniakos D, Wilson CL, Lahat A, Day CP, Burt A, Palmer A, Anstee QM, Grellscheid SN, Hoeijmakers JHJ, Barnhoorn S, Mann DA, Bird TG, Vermeij WP, Kirkland JL, Passos JF, von Zglinicki T, and Jurk D. 2017. Cellular senescence drives age-dependent hepatic steatosis. Nat Commun 8:15691. 10.1038/ncomms15691

Pardo V, Gonzalez-Rodriguez A, Muntane J, Kozma SC, and Valverde AM. 2015. Role of hepatocyte S6K1 in palmitic acid-induced endoplasmic reticulum stress, lipotoxicity, insulin resistance and in oleic acid-induced protection. Food Chem Toxicol 80:298-309. 10.1016/j.fct.2015.03.029

Pascal LE, True LD, Campbell DS, Deutsch EW, Risk M, Coleman IM, Eichner LJ, Nelson PS, and Liu AY. 2008. Correlation of mRNA and protein levels: cell type-specific gene expression of cluster designation antigens in the prostate. BMC Genomics 9:246. 10.1186/1471-2164-9-246

Pirc Marolt T, Kramar B, Bulc Rozman K, Suput D, and Milisav I. 2020. Aripiprazole reduces liver cell division. PLoS One 15:e0240754. 10.1371/journal.pone.0240754

Proell V, Carmona-Cuenca I, Murillo MM, Huber H, Fabregat I, and Mikulits W. 2007. TGF-beta dependent regulation of oxygen radicals during transdifferentiation of activated hepatic stellate cells to myofibroblastoid cells. Comp Hepatol 6:1. 10.1186/1476-5926-6-1

Rinella ME. 2015. Nonalcoholic fatty liver disease: a systematic review. JAMA 313:2263-2273. 10.1001/jama.2015.5370

Sanchez A, Alvarez AM, Benito M, and Fabregat I. 1996. Apoptosis induced by transforming growth factor-beta in fetal hepatocyte primary cultures: involvement of reactive oxygen intermediates. J Biol Chem 271:7416-7422. 10.1074/jbc.271.13.7416

Sancho P, Mainez J, Crosas-Molist E, Roncero C, Fernandez-Rodriguez CM, Pinedo F, Huber H, Eferl R, Mikulits W, and Fabregat I. 2012. NADPH oxidase NOX4 mediates stellate cell activation and hepatocyte cell death during liver fibrosis development. PLoS One 7:e45285. 10.1371/journal.pone.0045285

PeerJ reviewing PDF | (2021:07:63307:1:1:NEW 15 Sep 2021) 
309

310

311

312

313

314

315

316

317

318

319

320

321
Stone S, Jiang P, Dayananth P, Tavtigian SV, Katcher H, Parry D, Peters G, and Kamb A. 1995. Complex structure and regulation of the P16 (MTS1) locus. Cancer Res 55:2988-2994.

Torre LA, Bray F, Siegel RL, Ferlay J, Lortet-Tieulent J, and Jemal A. 2015. Global cancer statistics, 2012. CA Cancer J Clin 65:87-108. 10.3322/caac.21262

Tyagi E, Liu B, Li C, Liu T, Rutter J, and Grossman D. 2017. Loss of p16(INK4A) stimulates aberrant mitochondrial biogenesis through a CDK4/Rb-independent pathway. Oncotarget 8:55848-55862. 10.18632/oncotarget.19862

Wolff B, and Naumann M. 1999. INK4 cell cycle inhibitors direct transcriptional inactivation of NF-kappaB. Oncogene 18:2663-2666. 10.1038/sj.onc.1202617

Zhao R, Choi BY, Lee MH, Bode AM, and Dong Z. 2016. Implications of Genetic and Epigenetic Alterations of CDKN2A (p16(INK4a)) in Cancer. EBioMedicine 8:30-39. 10.1016/j.ebiom.2016.04.017 


\section{Figure 1}

\section{Gene expression}

p16 gene was determined in rat liver $(\mathrm{A})$ and cellular liver models: primary rat hepatocytes (B), immortalized mouse hepatocytes (IMHP) (C), human HepG2 cell line (D), Human embryonic kidney 293T (HEK 293T) cell line (E) and rat hepatoma FaO cell line (F). Rn: normalized reporter value (fluorescence of the reporter dye/fluorescence of a passive reference dye). 18S: blue, p16: red/green. 

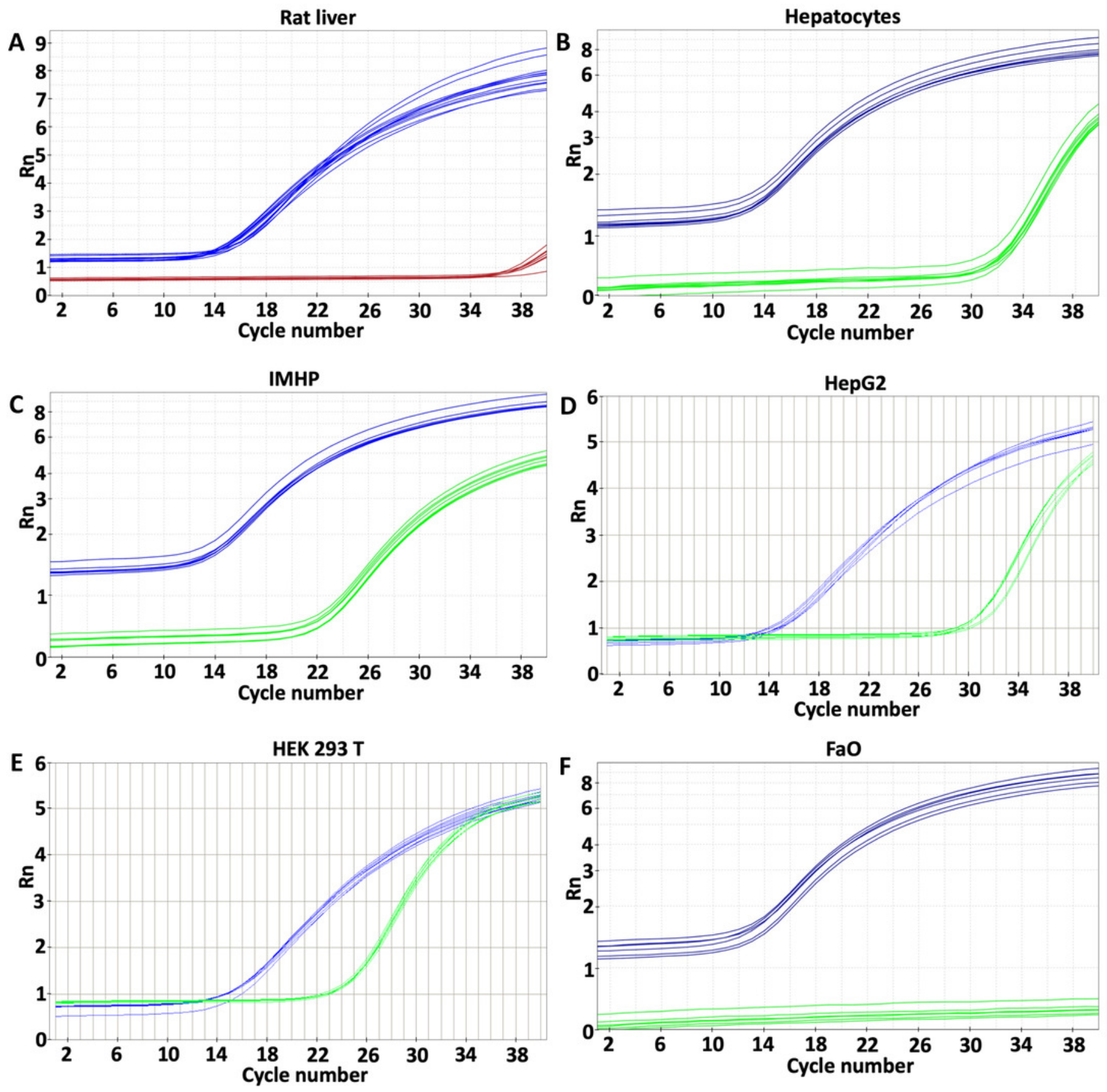


\section{Figure 2}

\section{Delta Ct values}

Mean delta Ct values of p16 and 18s RNA amplification in liver, hepatocytes and cell lines. Human embryonic kidney 293T (HEK 293T) cell line, immortalized mouse hepatocytes (IMHP), primary rat hepatocytes, $n=4$. Rat liver, HepG2 cell line, FaO cell line, $n=3$. Data are presented as the mean \pm standard deviation (SD) and are statistically significant $(P<0.1$ $x 10^{-14}$, one-way ANOVA; normal distribution of data: Brown-Forsythe test $P=0.56$ ). Pairwise differences between HEK293T and IMHP: P = 0.014; between HEK293T and any other cell type $\mathrm{P}<0.1 \times 10^{-14} ;$ IMHP and HepG2/hepatocytes/rat liver: $\mathrm{P}<0.1 \times 10^{-14}$; HepG2 and hepatocytes $P=1.3 \times 10^{-9}$; HepG2 and rat liver $P=9.2 \times 10^{-12}$; hepatocytes and rat liver $P=$ $7.3 \times 10^{-6}$ 


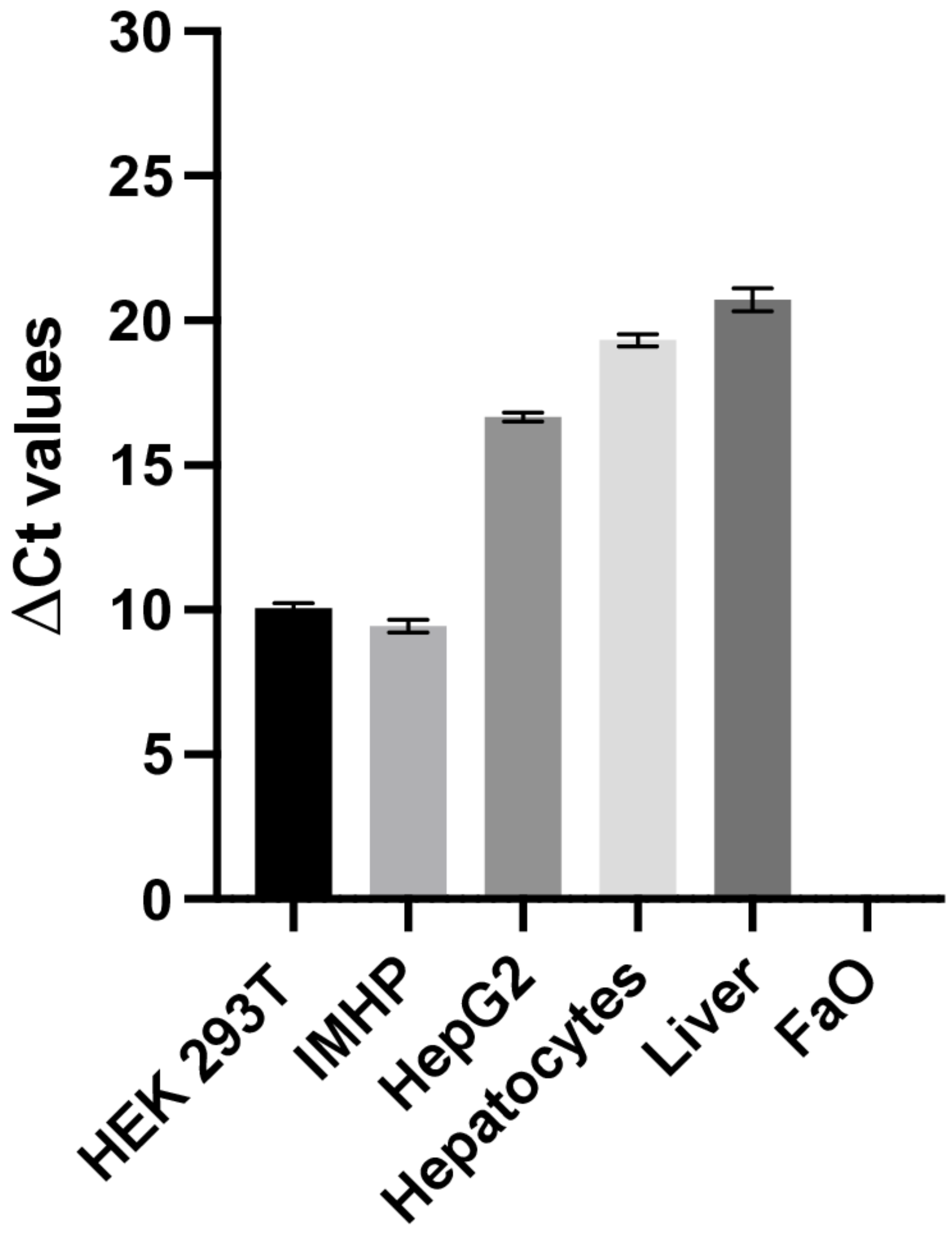




\section{Figure 3}

p16 protein levels

(A) Immunoblot was probed with a polyclonal antibody to p16. Each lane represents a lysate from one biological replicate. Underneath is a Coomassie-stained gel for loading control. (B) Densitometric analysis of protein levels normalized to a Coomassie- stained gel. The differences in p16 protein amounts are statistically significant $\left(P=4.0 \times 10^{-5}\right.$, one-way ANOVA; normal distribution of data: Brown-Forsythe test $P=0.19$ ). Pairwise comparison of the protein amounts: HEK293T to IMHP: $\mathrm{P}=8.9 \times 10^{-4}$, to HepG2: $\mathrm{P}=1.3 \times 10^{-3}$, to hepatocytes: $P=7.5 \times 10^{-5}$, to rat liver: $P=9.5 \times 10^{-5}$, to $\mathrm{FaO}: P=5.4 \times 10^{-5}$.
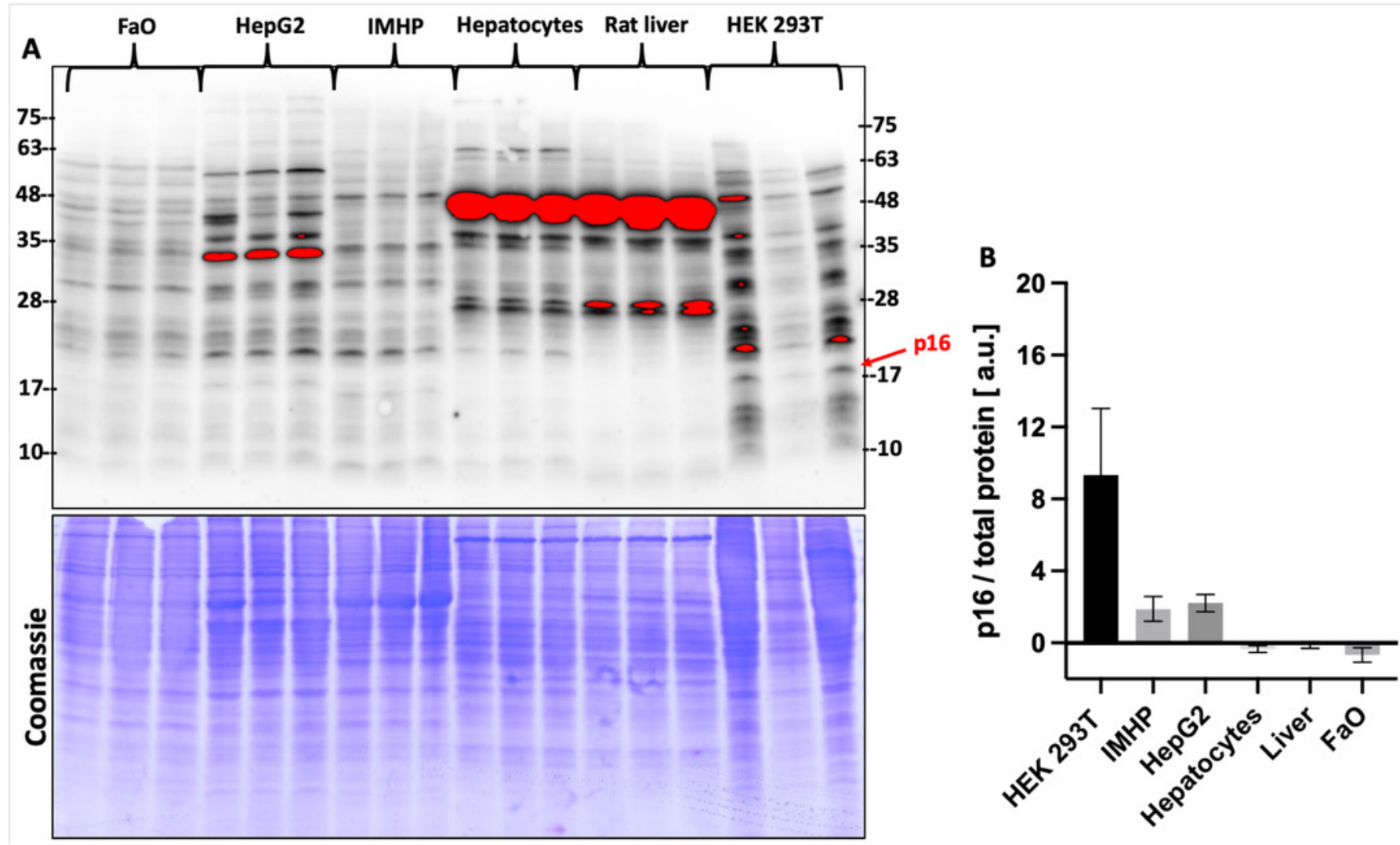


\section{Table $\mathbf{1}$ (on next page)}

Mean threshold cycle $(\mathrm{Ct})$ values and standard deviations of biological replicates of tested liver, hepatocytes and cell lines.

Human embryonic kidney (HEK 293T), immortalized mouse hepatocytes (IMHP), primary rat hepatocytes, $n=4$; rat liver, HepG2 and FaO cell line $n=3$. The differences between the cell lines are statistically significant $\left(P<0.1 \times 10^{-14}\right.$, one-way ANOVA; normal distribution of data: Brown-Forsythe test $\mathrm{P}=0.47$ ). 


\begin{tabular}{ccccccc}
\hline Cell model & Mean Ct values & SD & \multicolumn{2}{c}{ P values (Tukey's multiple comparisons test) } \\
& & & IMHP & HepG2 & Hepatocytes & Rat liver \\
\hline HEK 293T & 24.74 & 0.127 & 0.064 & $4.2 \times 10^{-12}$ & $2.5 \times 10^{-14}$ & $2.3 \times 10^{-14}$ \\
IMHP & 23.91 & 0.146 & $/$ & $6.3 \times 10^{-13}$ & $2.3 \times 10^{-14}$ & $2.3 \times 10^{-14}$ \\
HepG2 & 31.07 & 0.544 & $/$ & $/$ & $6.5 \times 10^{-6}$ & $3.5 \times 10^{-9}$ \\
Hepatocytes & 33.41 & 0.237 & $/$ & $/$ & $/$ & $6.3 \times 10^{-5}$ \\
Rat liver & 35.36 & 0.853 & $/$ & $/$ & $/$ & - \\
FaO & $\begin{array}{c}\text { No } \\
\text { amplification }\end{array}$ & & - & & \\
\hline
\end{tabular}

